\title{
Some fixed point theorems in locally $p$-convex spaces
}

\author{
Leila Gholizadeh ${ }^{1}$, Erdal Karapınar ${ }^{2 *}$ and Mehdi Roohi ${ }^{3}$
}

\section{"Correspondence:}

erdalkarapinar@yahoo.com; erdalkarapinar@atilim.edu.tr ${ }^{2}$ Department of Mathematics, Atllim University, Incek, Ankara 06836, Turkey

Full list of author information is available at the end of the article

\begin{abstract}
In this paper we investigate the existence of a fixed point of multivalued maps on almost $p$-convex and $p$-convex subsets of topological vector spaces. Our results extend and generalize some fixed point theorems on the topic in the literature, such as the results of Himmelberg, Fan and Glicksberg.

MSC: 46T99; 47H10; 54H25; 54E50; 55M20; 37C25

Keywords: fixed point theorems; locally convex space; $p$-convex set
\end{abstract}

\section{Introduction and preliminaries}

In nonlinear analysis, one of the dynamic research areas is investigation of existence of a fixed point of maps on convex sets and $p$-convex sets. Recently, a number of fixed point theorems have appeared on the setting of $p$-convex sets. For instance, Alimohammady et al. [1] extended the Markov-Kakutani fixed point theorem for compact $p$-star shaped subsets in topological vector spaces by using $p$-convex sets instead of convex sets, see also $[2,3]$. Further, in [4] authors achieved a fixed point theorem due to Park for a compact mapping on a $p$-star shaped subset of a topological vector space via Fan-KKM principle in a generalized convex space. In [5, 6], generalized versions of Brouwer and Kakutani fixed point theorems were characterized in the context of locally $p$-convex space.

On the other hand, in 1993 Park and Kim introduced the concept of generalized convex space, which extends many generalized convex structures on topological vector spaces [7]. This new concept, developed in connection with fixed point theory and KKM theory, generalizes topological vector spaces.

Maki [8] introduced the notion of minimal spaces which is a generalization of the concept of topological spaces (see also [9]). After these initial papers, many authors have paid attention to the subject and have published several results in this direction; see, e.g., [1013]. Very recently, Darzi et al. [14] introduced the notion of minimal generalized convex space as to extend the construction of the generalized convex space.

For the sake of completeness, we recall some basic definitions and fundamental results in the literature. All we need regarding topological vector spaces can be found in [15-18].

Let $U$ be a subset of a vector space $V$ and $x, y \in U$ and $0<p \leq 1$. Bayoumi [5] introduced the notion of arc segment joining $x$ and $y$, as follows:

$$
A_{x}^{y}=\left\{s^{\frac{1}{p}} x+t^{\frac{1}{p}} y: s+t=1\right\}=\left\{u x+v y: u^{p}+v^{p}=1\right\} .
$$

o 2013 Gholizadeh et al.; licensee Springer. This is an Open Access article distributed under the terms of the Creative Commons Attribution License (http://creativecommons.org/licenses/by/2.0), which permits unrestricted use, distribution, and reproduction in any medium, provided the original work is properly cited. 
A set $X$ in a vector space $V$ is said to be $p$-convex if $A_{x}^{y} \subseteq X$ for every $x, y \in X$. The $p$-convex hull of $X$ denoted by $C_{p}(X)$ is the smallest $p$-convex set containing $X$ [5]. Further, the closed $p$-convex hull of $X$ denoted by $\bar{C}_{p}(X)$ is the smallest closed $p$-convex set containing $X \subseteq E$, where $E$ is a topological vector space. Notice that if $p=1$ and $s+t=1$, then $A_{x}^{y}$ turns out to be the line segment joining $x$ and $y$. In this case, $C_{p}(X)$ and $\bar{C}_{p}(X)$ become the convex hull and the closed convex hull of $X$, respectively. For more details, we refer to, e.g., $[5,6$, 19-23] and references therein.

Let $X$ be a nonempty set. Then a family $\mathcal{M} \subseteq \mathcal{P}(X)$ is said to be a minimal structure on $X$ if $\emptyset, X \in \mathcal{M}$. Moreover, the pair $(X, \mathcal{M})$ is called a minimal space. The natural examples of minimal spaces can be listed as follows [8]: $\tau$, the collection of all semi-open sets $S O(X)$, the collection of all pre-open sets $P O(X)$, the collection of all $\alpha$-open sets $\alpha O(X)$ and the collection of all $\beta$-open sets $\beta O(X)$, where $(X, \tau)$ is a topological space. In a minimal space $(X, \mathcal{M})$, a set $A \in \mathcal{P}(X)$ is said to be an m-open set if $A \in \mathcal{M}$. Similarly, a set $B \in \mathcal{P}(X)$ is an $m$-closed set if $B^{c} \in \mathcal{M}$. Furthermore, $m$-interior and $m$-closure of a set $A$ are defined as follows:

$$
m-\operatorname{Int}(A)=\bigcup\{U: U \subseteq A, U \in \mathcal{M}\} \quad \text { and } \quad m-\operatorname{Cl}(A)=\bigcap\left\{F: A \subseteq F, F^{c} \in \mathcal{M}\right\} .
$$

For more details on minimal structure and minimal space, we refer the reader to, e.g., [8, $9,12-14,24,25]$.

The continuity of maps in a minimal space is defined as follows.

Definition 1.1 [25] Suppose that $(X, \tau)$ is a topological space, and also suppose that $(Y, \mathcal{N})$ is a minimal space. A function $f:(X, \tau) \longrightarrow(Y, \mathcal{N})$ is called $(\tau, m)$-continuous if $f^{-1}(U) \in \tau$ for any $U \in \mathcal{N}$.

Let $X$ and $Y$ be two nonempty sets and $\mathcal{P}(Y)$ be the set of all subsets of $Y$. A set-valued map or a set-valued function from $X$ into $Y$ is a function from $X$ to $\mathcal{P}(Y)$ that assigns an element $x$ of $X$ to a nonempty subset $T(x)$ of $Y$ and is denoted by $x \vdash T(x)$. The lower inverse of a point $y \in Y$ of a set-valued map $T$ is the set-valued map $T^{l}$ of $Y$ into $X$ defined by

$$
T^{l}(y)=\{x \in X: y \in T(x)\}
$$

Analogously, lower inverse of a subset of $B \subset Y$ is defined as

$$
T^{l}(B)=\{x \in X: T(x) \cap B \neq \emptyset\} .
$$

We note that $T^{l}(\emptyset)=\emptyset$. The set $\{x \in X: T(x) \subseteq B\}$ is the upper inverse of $B$ and is denoted by $T^{u}(B)$. A map $T$ is lower semicontinuous if $T^{l}(U)$ is open in $X$ for every open set $U \subseteq Y$. Similarly, a map $T$ is upper semicontinuous if for every open set $U \subseteq Y$, the set $T^{u}(U)$ is open in $X$.

A set-valued map $T: X \multimap Y$ is said to be closed if its graph, $\operatorname{Graph}(T)=\{(x, y): y \in T(x)\}$, is a closed subset of $X \times Y$. Also, $T$ is called compact if its range, $T(X)$, is contained in a compact subset of $Y$.

The notion of almost convex was introduced by Himmelberg [26]. A nonempty subset $B$ of a topological vector space $X$ is said to be almost convex if for any neighborhood $V$ 
of 0 and for any finite subset $\left\{b_{1}, \ldots, b_{n}\right\}$ of $B$, there exists a finite subset $\left\{x_{1}, \ldots, x_{n}\right\} \subseteq B$ such that $x_{i}-b_{i} \in V$ for each $i=1, \ldots, n$ and $c o\left(\left\{x_{1}, \ldots, x_{n}\right\}\right) \subseteq B$. It is clear that any convex subset is almost convex. Moreover, if we delete a certain subset of the boundary of a closed convex set, then we have an almost convex set. Another example of an almost convex set is the following: Let $C([0,1])$ be the Banach space of all continuous real functions defined on the unit interval $[0,1]$, and let $P([0,1])$ be a dense subset of all polynomials. Then any subset of $C([0,1])$ containing $P([0,1])$ is almost convex.

Let $A$ be a subset of a topological vector space $X$. A set-valued map $T: A \multimap A$ is said to have the (convexly) almost fixed point property if for every (convex) neighborhood $U$ of 0 in $X$, there exists a point $a_{U} \in A$ for which $a_{U} \in T\left(a_{U}\right)+U$ or $T\left(a_{U}\right) \cap\left(a_{U}+U\right) \neq \emptyset$.

Let $\langle D\rangle$ denote the set of all nonempty finite subsets of a set $D$, and let $\Delta_{n}$ be the $n$-simplex with vertices $e_{0}, e_{1}, \ldots, e_{n}, \Delta_{J}$ be the face of $\Delta_{n}$ corresponding to $J \in\langle A\rangle$, where $A \in\langle D\rangle$. For instance, if $A=\left\{a_{0}, a_{1}, \ldots, a_{n}\right\}$ and $J=\left\{a_{i_{0}}, a_{i_{1}}, \ldots, a_{i_{k}}\right\} \subseteq A$, then $\Delta_{J}=c o\left\{e_{i_{0}}, e_{i_{1}}, \ldots, e_{i_{k}}\right\}$. A minimal generalized convex space (briefly $M G$-convex space) $(X, D, \Gamma)$ consists of a minimal space $(X, \mathcal{M})$, a nonempty set $D$ and a set-valued map $\Gamma:\langle D\rangle \multimap X$ in which for $A \in\langle D\rangle$ with $n+1$ elements, there exists a $(\tau, m)$-continuous function $\phi_{A}: \Delta_{n} \longrightarrow \Gamma_{A}:=\Gamma(A)$ for which $J \in\langle A\rangle$ implies that $\phi_{A}\left(\Delta_{J}\right) \subseteq \Gamma_{J}$. If $\mathcal{M}=\tau$, then the notion of $M G$-convex space turns into G-convex space (see, e.g., [27]). On the other hand, suppose that $(X, \mathcal{M})$ is a minimal vector space which is not a topological vector space. Consider the set-valued map $\Gamma:\langle X\rangle \multimap X$ defined by $\Gamma\left(\left\{a_{0}, a_{1}, \ldots, a_{n}\right\}\right)=$ $\left\{\sum_{i=0}^{n} \lambda_{i} a_{i}: 0 \leq \lambda_{i} \leq 1, \sum_{i=0}^{n} \lambda_{i}=1\right\}$. Then $(X, \Gamma)$ is a minimal generalized convex space; of course, we know that $(X, \Gamma)$ is not a generalized convex space [14].

Definition 1.2 Suppose that $(X, D, \Gamma)$ is an $M G$-convex space. A set-valued map $F: D \multimap$ $X$ is called a $K K M$ set-valued map if $\Gamma_{A} \subseteq F(A)$ for any $A \in\langle D\rangle$.

We state two useful theorems of Alimohammady et al. [25] as follows.

Theorem 1.3 [25] Suppose that $(X, D, \Gamma)$ is an MG-convex space and $F: D \multimap X$ is a setvalued map satisfying

(a) for all $x \in D, F(x)=m-\mathrm{Cl}\left(A_{x}\right)$ for some $A_{x} \subseteq X$,

(b) $F$ is a KKM map.

Then $\{F(z): z \in D\}$ has the finite intersection property.

Further, if

(c) $\bigcap_{z \in N} F(z)$ is m-compact for some $N \in\langle D\rangle$,

then $\bigcap_{z \in D} F(z) \neq \emptyset$.

Theorem 1.4 [25] Suppose that $(X, D, \Gamma)$ is an $M G$-convex space and $F: D \multimap X$ is a setvalued map satisfying

(a) for all $x \in D, F(x)=m$-Int $\left(A_{x}\right)$ for some $A_{x} \subseteq X$,

(b) $F$ is a KKM map.

Then $\{F(z): z \in D\}$ has the finite intersection property.

In this paper we investigate the existence of a fixed point on the setting of locally $p$ convex spaces. In particular, we establish a generalized version of Alexandroff-Pasynkoff theorem. Furthermore, we present a generalization of the Himmelberg fixed point theorem. We also prove Fan-Glicksberg result for $p$-convex sets. 


\section{Main results}

We start this section with the following result which is inspired by Theorem 1.3 and Theorem 1.4.

Theorem 2.1 Suppose that $A$ is a subset of a topological vector space $X$ and $B$ is a nonempty subset of $A$ with $C_{p}(B) \subseteq A$. Also suppose that $F: B \multimap A$ is a set-valued map satisfying

(a) $F(b)$ is closed (resp. open) in $A$ for all $b \in B$,

(b) $C_{p}(N) \subseteq F(N)$ for each $N \in\langle B\rangle$.

Then $\{F(b): b \in B\}$ has the finite intersection property.

Proof Consider the set-valued map $\Gamma:\langle B\rangle \multimap A$ defined by

$$
\Gamma\left(\left\{b_{0}, b_{1}, \ldots, b_{n}\right\}\right)=\left\{\sum_{i=0}^{n} \lambda_{i} b_{i}: 0 \leq \lambda_{i} \leq 1, \sum_{i=0}^{n} \lambda_{i}^{p}=1\right\} .
$$

Since $C_{p}(B) \subseteq A$, the set-valued map $\Gamma$ is well defined. Condition (b) implies that $F$ is a KKM map. For each $N=\left\{b_{0}, b_{1}, \ldots, b_{n}\right\} \subseteq B$, let us define

$$
\begin{aligned}
& \phi_{N}: \Delta_{n} \longrightarrow \Gamma_{N}, \\
& \sum_{i=0}^{n} t_{i} e_{i} \mapsto \sum_{i=0}^{n}\left(t_{i}\right)^{\frac{1}{p}} b_{i} .
\end{aligned}
$$

Now, one can verify that $(A, B, \Gamma)$ is a $G$-convex space. The fact that $\{F(b): b \in B\}$ has the finite intersection property follows from Theorem 1.3 (resp. Theorem 1.4).

Theorem 2.2 Suppose that $A$ is a subset of an MG-convex space $(X, D, \Gamma),\left\{A_{0}, A_{1}, \ldots, A_{n}\right\}$ is a family of m-closure valued (resp. m-interior valued) subsets of $X$ such that $A \subseteq \bigcup_{i=0}^{n} A_{i}$, and also suppose that $N=\left\{z_{0}, z_{1}, \ldots, z_{n}\right\}$ is a family of points in $D$ in which $\Gamma(N) \subseteq A$. If $\Gamma\left(N \backslash\left\{z_{i}\right\}\right) \subseteq A_{i}$ for each $i=0,1, \ldots, n$, then $\bigcap_{i=0}^{n} A_{i} \neq \emptyset$.

Proof Set $C_{0}=\Gamma\left(N \backslash z_{n}\right)$ and for $i=1,2, \ldots, n$, let $C_{i}=\Gamma\left(N \backslash\left\{z_{i-1}\right\}\right)$. Consider the setvalued map $F: D \multimap X$ defined by $F\left(z_{0}\right)=A_{n}, F\left(z_{i}\right)=A_{i-1}$ for $i=1,2, \ldots, n$ and $F(z)=X$ for all $z \in D \backslash N$. We claim that $F$ is a KKM map. To see this, we note that $\Gamma(N) \subseteq A \subseteq$ $\bigcup_{i=0}^{n} A_{i}=F(N)$ and for any choice of a proper subset $\left\{z_{i_{0}}, z_{i_{1}}, \ldots, z_{i_{k}}\right\}$ of $N$ with $0 \leq k<n$ and $0 \leq i_{0}<\cdots<i_{k} \leq n$, one can see that

$$
\Gamma\left(\left\{z_{i_{0}}, z_{i_{1}}, \ldots, z_{i_{k}}\right\}\right) \subseteq C_{i_{j}} \subseteq A_{i_{j}-1}=F\left(z_{i_{j}}\right)
$$

for some $j \in\{0,1, \ldots, k\}$. Notice that $i_{j}=0$ if and only if $i_{j}-1=n$, and so $\Gamma\left(\left\{z_{i_{0}}, z_{i_{1}}, \ldots, z_{i_{k}}\right\}\right) \subseteq$ $\bigcup_{j=0}^{k} F\left(z_{i j}\right)$. The fact that $\bigcap_{i=0}^{n} A_{i} \neq \emptyset$ follows from Theorem 1.3 (resp. Theorem 1.4).

Remark 2.3 It should be noted that

(a) Theorem 1.3 and Theorem 1.4 are extended versions of the corresponding results in $[14,24]$, and hence they are generalizations of Theorem 1 in $[27,28]$ and Ky Fan's lemma [29],

(b) Theorem 2.2 for closed (open) subsets of a topological vector space goes back to Park [30] and it is an extended version of Alexandroff-Pasynkoff theorem [31]. 
Definition 2.4 A nonempty subset $B$ of a topological vector space $X$ is said to be almost $p$-convex if for any neighborhood $V$ of 0 and for any finite subset $\left\{b_{1}, \ldots, b_{n}\right\}$ of $B$, there exists a finite subset $\left\{x_{1}, \ldots, x_{n}\right\} \subseteq B$ such that $x_{i}-b_{i} \in V$ for each $i=1, \ldots, n$ and $C_{p}\left(\left\{x_{1}, \ldots, x_{n}\right\}\right) \subseteq B$.

Example 2.5 It is easy to see that any $p$-convex subset of a topological vector space $X$ is almost $p$-convex. If we delete a certain subset of the boundary of a closed $p$-convex set, then we have an almost $p$-convex set.

Definition 2.6 Let $A$ be a subset of a topological vector space $X$. A set-valued map $T$ : $A \multimap A$ is said to have the $p$-convexly almost fixed point property if for every $p$-convex neighborhood $U$ of 0 in $X$, there exists a point $a_{U} \in A$ for which $a_{U} \in T\left(a_{U}\right)+U$ or $T\left(a_{U}\right) \cap$ $\left(a_{U}+U\right) \neq \emptyset$.

Theorem 2.7 Let $A$ be a subset of a topological vector space $X$ and $B$ be an almost $p$ convex dense subset of $A$. Suppose that $T: A \multimap X$ is a lower (resp. upper) semicontinuous set-valued map such that $T(b)$ is $p$-convex for all $b \in B$, and also suppose that there is a precompact subset $K$ of $A$ such that $T(b) \cap K \neq \emptyset$ for all $b \in B$. Then $T$ has the $p$-convexly almost fixed point property.

Proof Suppose that $U$ is a $p$-convex neighborhood of 0 and suppose that $T$ is lower semicontinuous. There is a symmetric open neighborhood $V$ of 0 for which $\bar{V}+\bar{V} \subseteq U$. Since $K$ is precompact, so there are $x_{0}, x_{1}, \ldots, x_{n}$ in $K$ for which $K \subseteq \bigcup_{i=0}^{n}\left(x_{i}+V\right)$. By using the fact that $B$ is almost $p$-convex and dense in $A$, we find $D=\left\{b_{0}, b_{1}, \ldots, b_{n}\right\} \subseteq B$ for which $b_{i}-x_{i} \in V$ for all $i \in\{0,1, \ldots, n\}$ and also $C=C_{p}(D) \subseteq B$. Since $T$ is lower semicontinuous, the set $F\left(b_{i}\right):=\left\{c \in C: T(c) \cap\left(x_{i}+V\right)=\emptyset\right\}$ is closed in $C$ for each $i \in\{0, \ldots, n\}$. Regarding $\emptyset \neq T(c) \cap K \subseteq T(c) \cap \bigcup_{i=0}^{n}\left(x_{i}+V\right)$, we have $\bigcap_{i=0}^{n} F\left(b_{i}\right)=\emptyset$. Now, Theorem 2.1 implies that there is $N=\left\{b_{i_{0}}, b_{i_{1}}, \ldots, b_{i_{k}}\right\} \in\langle D\rangle$ and $x_{U} \in C_{p}(N) \subseteq B$ for which $x_{U} \notin F(N)$, and so $T\left(x_{u}\right) \cap\left(x_{i_{j}}+\bar{V}\right) \neq \emptyset$ for all $j \in\{0,1, \ldots, k\}$. Both $b_{i}-x_{i} \in V$ and $\bar{V}+\bar{V} \subseteq U$ imply that $x_{i_{j}}+\bar{V} \subseteq b_{i_{j}}+U$, which implies that $T\left(x_{U}\right) \cap\left(b_{i_{j}}+U\right) \neq \emptyset$. Therefore

$$
N \subseteq M:=\left\{c \in C: T\left(x_{U}\right) \cap(c+U) \neq \emptyset\right\}
$$

$C, T\left(x_{U}\right)$ and $U$ are $p$-convex and hence $M$ is $p$-convex. Consequently, $x_{U} \in M$, which implies that $T\left(x_{U}\right) \cap\left(x_{U}+U\right) \neq \emptyset$; i.e., $T$ has the $p$-convexly almost fixed point property. Finally, for the case that $T$ is upper semicontinuous, we note that $F\left(b_{i}\right):=\{c \in C: T(c) \cap$ $\left.\left(x_{i}+\bar{V}\right)=\emptyset\right\}$ is open in $C$ for each $i \in\{0, \ldots, n\}$. The rest of the proof is similar to the proof of the case that $T$ is l.s.c. Regarding the analogy, we skip the proof.

Corollary 2.8 Let $A$ be a p-convex subset of a topological vector space $X$, and let $T: A \multimap X$ be a lower (resp. upper) semicontinuous set-valued map such that $T(a)$ is $p$-convex for all $a \in A$. Suppose that there is a precompact subset $K$ of $A$ such that $T(a) \cap K \neq \emptyset$ for all $a \in A$.

Then $T$ has the p-convexly almost fixed point property.

Proof It is sufficient to take $A=B$ in Theorem 2.7.

Corollary 2.9 Let $A$ be a subset of a topological vector space $X$, and let $B$ be an almost $p$-convex dense subset of $A$. Suppose that $T: A \multimap X$ is a set-valued map satisfying 
(a) $T^{l}(x)\left(\right.$ resp. $\left.T^{u}(x)\right)$ is open for all $x \in X$,

(b) $T(b)$ is $p$-convex for all $b \in B$,

(c) there is a precompact subset $K$ of $A$ such that $T(b) \cap K \neq \emptyset$ for all $b \in B$.

Then $T$ has the p-convexly almost fixed point property.

Proof It is clear that (a) implies that $T$ is a lower (resp. upper) semicontinuous set-valued map and hence $T$ has the $p$-convexly almost fixed point property by Theorem 2.7 .

Corollary 2.10 Let $A$ be a p-convex subset of a topological vector space $X$, and let $T: A \multimap$ $X$ be a compact set-valued map satisfying the following conditions:

(a) $T^{l}(x)\left(\right.$ resp. $\left.T^{u}(x)\right)$ is open for all $x \in X$,

(b) $T(a)$ is nonempty and $p$-convex for all $a \in A$.

Then $T$ has the p-convexly almost fixed point property.

Proof Consider $A=B$, it is easy to see that all the conditions of Corollary 2.9 are satisfied.

Remark 2.11 It should be noted that

(a) Corollary 2.8 for a lower semicontinuous set-valued map on a locally convex Hausdorff topological vector space goes back to Ky Fan [32]. Corollary 2.8 for a single-valued map might be regarded as a generalization of the Thychonoff fixed point theorem to a noncompact (or precompact) convex set [32]. Also, Lassonde obtained Corollary 2.8 for a compact upper semicontinuous set-valued map with nonempty convex values [33].

(b) Convex versions of Theorem 2.7, Corollary 2.9 and Corollary 2.10 are due to Park [30].

Theorem 2.12 Suppose that $A$ is a subset of a locally $p$-convex space $X$ and $B$ is an almost $p$-convex dense subset of $A$. Suppose that $T: A \multimap A$ satisfies the following:

(a) $T$ is compact upper semicontinuous,

(b) $T(a)$ is closed for all $a \in A$,

(c) $T(b)$ is nonempty $p$-convex for all $b \in B$.

Then $T$ has a fixed point.

Proof Since all the conditions of Theorem 2.7 are satisfied and since $X$ is a locally $p$-convex space, $T$ has the almost fixed point property. Then, for an arbitrary neighborhood $U$ of 0 , there exist $a_{U}$ and $b_{U}$ in $A$ for which $b_{U} \in T\left(a_{U}\right) \cap\left(a_{U}+U\right)$. Since $T$ is compact, we conclude that there is $a_{0} \in \overline{T(A)} \subseteq A$ in which the net $b_{U} \longrightarrow a_{0}$. Because $X$ is Hausdorff, $a_{U} \longrightarrow a_{0}$. Since $T$ is an upper semicontinuous set-valued map with closed values, $\operatorname{Graph}(T)$ is closed. Consequently, $a_{0}$ is a fixed point of $T$.

Corollary 2.13 Suppose that $A$ is a p-convex subset of a locally p-convex space X. Suppose that $T: A \multimap A$ satisfies the following:

(a) $T$ is compact upper semicontinuous,

(b) $T(a)$ is closed for all $a \in A$,

(c) $T(a)$ is nonempty $p$-convex for all $a \in A$.

Then $T$ has a fixed point. 
Theorem 2.14 Suppose that $A$ is a $p$-convex subset of a locally $p$-convex space $X$. Suppose that $T: A \multimap A$ satisfies the following:

(a) $T$ is compact and closed,

(b) $T$ has the almost fixed point property.

Then $T$ has a fixed point.

Proof Suppose that $\mathcal{U}$ is the family of neighborhoods of 0 in $X$. For any element $U$ of $\mathcal{U}$, since $T$ has the almost fixed point property, so there exist $a_{U}, b_{U} \in A$ for which $b_{U} \in T\left(a_{U}\right)$ and $b_{U} \in a_{U}+U$. Now, consider the nets $\left\{a_{U}\right\}$ and $\left\{b_{U}\right\}$. By (a) we have $\overline{T(A)}$ is compact and hence $\left\{b_{U}\right\}$ has a subnet converging to $b_{0}$. We may assume that $b_{U} \longrightarrow b_{0}$. Since $X$ is Hausdorff, there is a subnet of $a_{U}$ converging to $b_{0}$. The fact that $b_{0} \in T\left(b_{0}\right)$ follows from $\left(a_{U}, b_{U}\right) \in \operatorname{Graph}(T)$ and the fact that $\operatorname{Graph}(T)$ is closed.

Corollary 2.15 Suppose that $A$ is a $p$-convex subset of a locally $p$-convex space $X$ and that $T: A \multimap A$ satisfies the following:

(a) $T$ is compact and closed,

(b) $T^{l}(x)\left(\right.$ resp. $\left.T^{u}(x)\right)$ is open for all $x \in X$,

(c) $T(a)$ is nonempty and $p$-convex for all $a \in A$.

Then $T$ has a fixed point.

Proof It is an immediate consequence of Corollary 2.10 and Theorem 2.14.

Remark 2.16 Corollary 2.13 is a generalization of the main results of Himmelberg [26]. Theorem 2.12 for $p=1$ goes back to Park [30]. Further, Theorem 2.14 for $p=1$ is an extension of Himmelberg's theorem (see, e.g., [34]).

For a set-valued map $T: X \multimap Y$, set $T_{B}=\{x \in X: x \in T(x)+B\}$ for $B \subseteq Y$.

Lemma 2.17 Suppose that $A$ is a $p$-convex subset of a topological vector space $X$, and also suppose that $\mathcal{U}$ is a fundamental system of open neighborhoods of 0 . Then, for a set-valued map $T: A \multimap X$, the following are equivalent:

(a) If $a \in A$ satisfies $a \notin T(a)+U$ for some $U \in \mathcal{U}$, then

$$
a \notin \mathrm{Cl}\left(\left\{a \in A: a \in T(a)+C_{p}(V)\right\}\right) \text { for some } V \in \mathcal{U} \text {, }
$$

(b) $\bigcap_{U \in \mathcal{U}} T_{U}=\bigcap_{U \in \mathcal{U}} \overline{T_{C_{p}(U)}}$.

Proof It is straightforward.

Remark 2.18 The conditions (a) and (b) considered in Lemma 2.17 for $p=1$ are due to Kim [35].

Theorem 2.19 Let $A$ be a p-convex compact subset of a topological vector space $X$, and let $T: A \multimap X$ be a mapping satisfying the following conditions:

(a) $T$ has the p-convexly almost fixed point property,

(b) $\bigcap_{U \in \mathcal{U}} T_{U}=\bigcap_{U \in \mathcal{U}} \overline{T_{C_{p}(U)}}$.

Then $\bar{T}$ has a fixed point. 
Proof Suppose that $\mathcal{U}$ is a fundamental system of open neighborhoods of 0 . Since $T$ has the $p$-convexly almost fixed point property, for any $U \in \mathcal{U}$, there is an $a_{U} \in A$ such that $a_{U} \in T\left(a_{U}\right)+C_{p}(U)$. Hence, $T_{C_{p}(U)} \neq \emptyset$ for each $U \in \mathcal{U}$. Now, since $\mathcal{U}$ is a fundamental system of open neighborhoods of 0 , we deduce that for any $U, V \in \mathcal{U}$, there is $W \in \mathcal{U}$ such that

$$
T_{C_{p}(U)} \cap T_{C_{p}(V)} \supseteq T_{C_{p}(U \cap V)} \supseteq T_{C_{p}(W)} \neq \emptyset .
$$

Therefore $\left\{T_{C_{p}(U)}: U \in \mathcal{U}\right\}$ has the finite intersection property. It follows from the compactness of $A$ that $\bigcap_{U \in \mathcal{U}} \overline{T_{C_{p}(U)}} \neq \emptyset$. Therefore, by the condition (b) there is an $a_{0} \in A$ for which $a_{0} \in \bigcap_{U \in \mathcal{U}} T_{U}$, that is, $a_{0} \in T\left(a_{0}\right)+\mathcal{U}$ for all $U \in \mathcal{U}$. Regarding $\bigcap_{U \in \mathcal{U}}\left(T\left(a_{0}\right)+\mathcal{U}\right)=$ $\overline{T\left(a_{0}\right)}$, we derive that $\bar{T}$ has a fixed point.

Corollary 2.20 Let $A$ be a $p$-convex compact subset of a topological vector space $X$, and let $T: A \multimap X$ be a mapping such that

(a) $T$ has the p-convexly almost fixed point property,

(b) $\bigcap_{U \in \mathcal{U}} T_{U}=\bigcap_{U \in \mathcal{U}} \overline{T_{C_{p}(U)}}$,

(c) Thas closed values.

Then $T$ has a fixed point.

Corollary 2.21 Let $A$ be a p-convex compact subset of a topological vector space $X$, and let $T: A \multimap A$ be a mapping such that

(a) $T$ is lower (resp. upper) semicontinuous,

(b) $T$ has $p$-convex values,

(c) $\bigcap_{U \in \mathcal{U}} T_{U}=\bigcap_{U \in \mathcal{U}} \overline{T_{C_{p}(U)}}$.

Then $\bar{T}$ has a fixed point.

Proof Since $A$ is a $p$-convex and compact, by (a) and (b) one can see that all the conditions of Corollary 2.8 hold. Then $T$ has the $p$-convexly almost fixed point property. The fact that $\bar{T}$ has a fixed point follows from Theorem 2.19.

Corollary 2.22 Let $A$ be a p-convex compact subset of a topological vector space $X$, and let $T: A \multimap A$ be a mapping satisfying the following conditions:

(a) $T$ is lower (resp. upper) semicontinuous,

(b) $T$ has closed $p$-convex values,

(c) $\bigcap_{U \in \mathcal{U}} T_{U}=\bigcap_{U \in \mathcal{U}} \overline{T_{C_{p}(U)}}$.

Then $T$ has a fixed point.

Remark 2.23 Corollary 2.22 for $p=1$ and lower semicontinuous set-valued maps goes back to Kim [35] and Park [36], and also this result for $p=1$ and upper semicontinuous set-valued maps is due to Huang and Jeng [37].

Theorem 2.24 Let $A$ be a compact $p$-convex subset of a locally $p$-convex space $X$, and let the set-valued map $T: A \multimap A$ be a mapping such that

(a) $T$ has the p-convexly almost fixed point property,

(b) $T$ is a closed set-valued map.

Then $T$ has a fixed point. 
Proof Suppose that $\mathcal{U}$ is a fundamental system of $p$-convex open neighborhoods of 0 . Then, for any $U \in \mathcal{U}$, there is $V \in \mathcal{U}$ for which $V \subseteq \bar{V} \subseteq U$. Now, we claim that $T_{C_{p}(\bar{V})}=T_{\bar{V}}$ is closed. To see this, let $a \in \overline{T_{\bar{V}}}$. There is a net $\left\{a_{i}: i \in I\right\} \subseteq T_{\bar{V}}$ for which $a_{i} \longrightarrow a$. Then, for each $i \in I$, there exists $b_{i} \in T\left(a_{i}\right)$ in which $a_{i}-b_{i} \in \bar{V}$. Since $T$ is compact and since $b_{i} \in T(A)$, so one can assume that $b_{i} \longrightarrow b$ for some $b \in \overline{T(A)}$, and so $a-b \in \bar{V} . b \in T(a)$, because $T$ is closed. Therefore,

$$
a \in(b+\bar{V}) \cap A \subseteq(T(a)+\bar{V}) \cap A ;
$$

i.e., $a \in T_{\bar{V}}$. Finally, since $T_{\bar{V}}$ is closed, and $V \subseteq \bar{V} \subseteq U$, so

$$
\bigcap_{U \in \mathcal{U}} T_{C_{p}(U)}=\bigcap_{U \in \mathcal{U}} T_{U}=\bigcap_{V \in \mathcal{U}} T_{\bar{V}}=\bigcap_{V \in \mathcal{U}} \overline{T_{\bar{V}}}=\bigcap_{U \in \mathcal{U}} \overline{T_{U}}=\bigcap_{U \in \mathcal{U}} \overline{T_{C_{p}(U)}} .
$$

Consequently, all the conditions of Corollary 2.20 hold and hence $T$ has a fixed point.

Remark 2.25 Theorem 2.24 is a generalization of the Fan-Glicksberg theorem [38, 39] and its convex version can be found in [34]. Notice also that Theorem 2.24 can be derived from Theorem 2.14.

\section{Competing interests}

The authors declare that there is no conflict of interests regarding the publication of this article.

\section{Authors' contributions}

All authors contributed equally and significantly in writing this article. All authors read and approved the final manuscript.

\section{Author details}

${ }^{1}$ Department of Mathematics, Islamic Azad University, Sari Branch, Sari, Iran. ${ }^{2}$ Department of Mathematics, Atılım University, İncek, Ankara 06836, Turkey. ${ }^{3}$ Department of Mathematics, Faculty of Sciences, Golestan University, P.O. Box. 155, Gorgan, Iran.

Received: 19 July 2013 Accepted: 28 October 2013 Published: 25 November 2013

\section{References}

1. Alimohammady, M, Roohi, M, Gholizadeh, L: On the Markov-Kakutani's fixed point theorem. Sci. Stud. Res. Ser. Math. Inform. 19(1), 17-22 (2009)

2. Kim, WK: Some application of the Kakutani fixed point theorem. J. Math. Anal. Appl. 121, 119-122 (1987)

3. Park, S: Fixed points on star-shaped sets. Nonlinear Anal. Forum 6(2), 275-279 (2001)

4. Alimohammady, M, Roohi, M, Gholizadeh, L: Remarks on the fixed points on star-shaped sets. Kochi J. Math. 3 , 109-116 (2008)

5. Bayoumi, A: Foundations of Complex Analysis in Nonlocally Convex Spaces. North-Holland Mathematics Studies, vol. $193(2003)$

6. Bayoumi, A: Generalized Brouwer's and Kakutani's fixed points theorems in non-locally convex spaces. To appear

7. Park, S, Kim, H: Admissible classes of multifunctions on generalized convex spaces. Proc. Coll. Nature Sci. SNU 18, 1-21 (1993)

8. Maki, H: On generalizing semi-open sets and preopen sets. In: Meeting on Topological Spaces Theory and Its Application. August (1996), 13-18

9. Maki, H, Umehara, J, Noiri, T: Every topological space is pre $T_{1}$. Mem. Fac. Sci. Kochi Univ. Ser. a Math. 17, 33-42 (1996)

10. Alimohammady, M, Roohi, M: Extreme points in minimal spaces. Chaos Solitons Fractals 39(3), 1480-1485 (2009)

11. Alimohammady, M, Roohi, M: Fixed point in minimal spaces. Nonlinear Anal. Model. Control 10(4), 305-314 (2005)

12. Alimohammady, M, Roohi, M: Linear minimal space. Chaos Solitons Fractals 33(4), 1348-1354 (2007)

13. Popa, V, Noiri, T: On M-continuous functions. Anal. Univ. "Dunarea Jos"-Galati, Ser. Mat. Fiz. Mec. Teor. Fasc. II 18(23), 31-41 (2000)

14. Darzi, R, Delavar, MR, Roohi, M: Fixed point theorems in minimal generalized convex spaces. Filomat 25(4), 165-176 (2011)

15. Adasch, N, Ernst, B, Keim, D: Topological Vector Spaces, the Theory Without Convexity Conditions. Lecture Notes in Mathematics, vol. 69. Springer, Berlin (1978)

16. Kladelburg, Z, Radenovic, S: Subspaces and Quotients of Topological and Ordered Vector Spaces. University of Novi Sad. Institute of Mathematics, Novi Sad (1997)

17. Kothe, G, Topological Vector Spaces, I. Springer, New York (1969) 
18. Schaefer, HH: Topological Vector Spaces. Springer, Berlin (1970)

19. Bastero, J, Bernues, J, Pena, A: The theorems of Caratheodory and Gluskin for $0<p<1$. Proc. Am. Math. Soc. 123, 141-144 (1995)

20. Bernues, J, Pena, A: On the shape of $p$-convex hulls, $0<p<1$. Acta Math. Hung. 74(4), 345-353 (1997)

21. Alimohammady, M, Roohi, M, Delavar, MR: Transfer closed and transfer open multimaps in minimal spaces. Chaos Solitons Fractals 40(3), 1162-1168 (2009)

22. Knaster, B, Kuratowski, K, Mazurkiewicz, S: Ein Beweis des fixpunktsatzes fur $n$-dimensionale simplexe. Fundam. Math. 14, 132-137 (1929)

23. Shih, M-H, Tan, K-K: Covering theorem of convex sets related to fixed-point theorems. In: Lin, B-L, Simons, S (eds.) Nonlinear and Convex Analysis-Proc. in Honor of Ky Fan, pp. 235-244. Marcel Dekker, New York (1987)

24. Alimohammady, M, Roohi, M, Delavar, MR: Knaster-Kuratowski-Mazurkiewicz theorem in minimal generalized convex spaces. Nonlinear Funct. Anal. Appl. 13(3), 483-492 (2008)

25. Alimohammady, M, Roohi, M, Delavar, MR: Transfer closed multimaps and Fan-KKM principle. Nonlinear Funct. Anal. Appl. 13(4), 597-611 (2008)

26. Himmelberg, CJ: Fixed points of compact multifunctions. J. Math. Anal. Appl. 38, 205-207 (1972)

27. Park, S: Remarks on topologies of generalized convex spaces. Nonlinear Funct. Anal. Appl. 5(2), 67-79 (2000)

28. Park, S: Ninety years of the Brouwer fixed point theorem. Vietnam J. Math. 27, 193-232 (1999)

29. Fan, K: A generalization of Tychonoff's fixed point theorem. Math. Ann. 142, 305-310 (1961)

30. Park, S: The Knaster-Kuratowski-Mazurkiewicz theorem and almost fixed points. Topol. Methods Nonlinear Anal. 16, 195-200 (2000)

31. Alexandroff, P, Pasynkoff, B: Elementary proof of the essentiality of the identity mappings of a simplex. Usp. Mat. Nauk 12, 175-179 (1957) (Russian)

32. Fan, K: Covering properties of convex sets and fixed point theorems in topological vector spaces. In: Symposium on Infinite Dimensional Topology. Ann. of Math. Studies, vol. 69, pp. 79-92 (1972)

33. Lassonde, M: Sur le principle KKM. C. R. Acad. Sci. Paris 310, 573-576 (1990)

34. Park, S: Remarks on fixed point theorems for new classes of multimaps. J. Natl. Acad. Sci., Rep. Korea 43, 1-20 (2004)

35. Kim, WK: A fixed point theorem in a Hausdorff topological vector space. Comment. Math. Univ. Carol. 36, 33-38 (1995)

36. Park, S: Fixed points theorems for new classes of multimaps. Acta Math. Hung. 81, 155-161 (1998)

37. Huang, Y-Y, Jeng, J-C: Fixed points theorems of the Park type in S-KKM class. Nonlinear Anal. Forum 5, 51-59 (2000)

38. Park, S: A unified fixed point theory of multimaps on topological vector spaces. J. Korean Math. Soc. 35, 803-829 (1998) Corrections, ibid. 36, 829-832 (1999)

39. Park, S: Some coincidence theorems on acyclic multifunctions and applications to KKM theory. In: Tan, K-K (ed.) Fixed Point Theory and Applications, pp. 248-277. World Scientific, River Edge (1992)

doi:10.1186/1687-1812-2013-312

Cite this article as: Gholizadeh et al.: Some fixed point theorems in locally $p$-convex spaces. Fixed Point Theory and Applications 2013 2013:312.

\section{Submit your manuscript to a SpringerOpen ${ }^{\ominus}$ journal and benefit from:}

- Convenient online submission

- Rigorous peer review

- Immediate publication on acceptance

- Open access: articles freely available online

- High visibility within the field

- Retaining the copyright to your article 